Pacific Journal of Mathematics

SETS OF UNIQUENESS AND SYSTEMS OF INEQUALITIES 


\section{SETS OF UNIQUENESS AND SYSTEMS OF INEQUALITIES HAVING A UNIQUE SOLUTION}

\section{J. H. B. KEMPERMAN}

Suppose a number of $\mathrm{X}$-ray pictures is taken of the same object, but from different directions. One typically likes to know to what degree the pictures determine the object and exactly when an object is uniquely determined. Replacing picture taking by projections, that is, images relative to specified mappings, these same problems are easily formulated for higher dimensions and even for abstract spaces. The objects on hand might be data structures.

With this general framework, starting from an arbitrary but fixed collection of mappings, we study a new and very useful class of objects (sets) each of which is uniquely determined by its projections. In the process, we disprove a previously conjectured characterization of uniqueness relative to the one-dimensional projections in $R^{n}$. For all situations where the underlying space is finite, a complete and rather simple characterization of uniqueness is obtained.

1. Introduction. Suppose an X-ray picture is made of an object $S$ in $R^{3}$ of uniform unit density. This corresponds to the creation of an image of $S$ on a photographic plate $Y$ by means of a central or parallel projection $\pi: R^{3} \rightarrow Y$. The darkness of the image at $y \in Y$ is directly related to the length $|L \cap S|$ of the part of $L$ inside $S$, with $L$ as the straight line $L=\pi^{-1}\{y\}=\left\{x \in R^{3}: \pi x=y\right\}$. Hence, having such a $\pi$-photograph of $S$ is equivalent to knowing the precise value $\lambda(S \cap A)$, for each set of the form $A=\pi^{-1} B=\left\{x \in R^{3}: \pi x \in B\right\}$. Here, $B$ can be any subset of $Y$ while $\lambda$ is Lebesgue measure on $R^{3}$.

Given any finite class of such photographic maps $\pi_{j}: R^{3} \rightarrow Y_{j}$, we would like to know exactly what subsets $S$ of $R^{3}$ are such that $S$ is uniquely determined by its set of images.

The subset $S$ of $R^{3}$ can be identified with the measure $\mu_{s}$ on $R^{3}$ defined by $\mu_{S}(A)=\lambda(S \cap A)$. Thus $\mu_{s}$ has its density relative to Lebesgue measure $\lambda$ equal to the function $1_{s}(x)$ on $X$ (1 on $S$ and 0 on its complement $S^{c}$ ). If $\pi: R^{3} \rightarrow Y$ is any map then the $\pi$ projection of $\mu_{s}$ onto $Y$ (also nonchalantly called the $\pi$-projection of $S$ ) is the mass distribution (measure) $\pi \mu_{S}$ on $Y$, whose mass inside any subset of $B$ of $Y$ equals $\mu_{s}(A)=\lambda(S \cap A)$ with $A=\pi^{-1} B=$ $\left\{x \in R^{3}: \pi x \in B\right\}$. Knowing the $\pi$-photograph of $S$ is the same as knowing the projection $\pi \mu_{s}$ of $\mu_{s}$. 
More generally, let $X$ be any space supplied with a reference measure $\lambda$. Let further $\left\{\pi_{j}, j \in J\right\}$ be a fixed finite or infinite collection of maps $\pi_{j}: X \rightarrow Y_{j}$. A subset $S$ of $X$ will be identified with the associated measure $d \mu_{s}=1_{s} d \lambda$ on $X$, (carried by $S$ and having a uniform density there). In particular, subsets $S$ and $T$ equal a.e. [ $\lambda$ ] are identified. The $\pi_{j}$-projection of $S$ is defined as the measure $\pi_{j} \mu_{s}$ on $Y_{j}$ whose mass $\left(\pi_{j} \mu_{s}\right)(B)$ inside any subset $B$ of $Y_{j}$ equals $\mu_{s}(A)=\lambda(S \cap A)$ with $A=\left(\pi_{j}\right)^{-1} B=\left\{x \in X: \pi_{j} x \in B\right\}$.

In the literature, much attention is given to the following situation, which we will refer to as the classical case. Here, $X=R^{n}$ supplied with $n$-dimensional Lebesgue measure $\lambda=m_{n}$ and projections $\pi_{1}, \ldots, \pi_{n}$, with $\pi_{j}$ as the orthogonal projection of $R^{n}$ onto the $j$ th coordinate axis. Relative to this classical case, knowing the $\pi_{j}$-projections of a subset $S$ of $R^{n}(j=1, \ldots, n)$ is the same as knowing the $(n-1)$-dimensional volume of the cross section of $S$ with each hyperplane $x_{j}=c$ perpendicular to one of the coordinate axes.

Going back to the general case, we will be interested in the class of subsets $S$ of $X$ that are uniquely determined by the associated collection $\left\{\pi_{j} \mu_{s}: j \in J\right\}$ of projections of $d \mu_{s}=1_{s} d \lambda$. It is always assumed that $\lambda(S)<\infty$. We say that $S$ is a weak set of uniqueness (relative to $X, \lambda$ and the $\pi_{j}$ ) if there is no other set $T$ such that $\pi_{j} \mu_{T}=\pi_{j} \mu_{s}$, for all $j \in J$. Here, we identify sets which are equal a.e. [ $[\lambda]$. And we call $S$ a (strong) set of uniqueness if besides $\mu_{s}$ there does not exist any measure $\mu$ of the form $d \mu=\phi d \lambda$, with $\phi: X \rightarrow[0,1]$, such that $\pi_{j} \mu=\pi_{j} \mu_{s}$, for all $j \in J$.

These notions are distinct if $X$ is discrete, see [2]. On the other hand, the two notions of uniqueness coincide in the important case that $X=R^{n}$ with $\lambda$ of the form $\lambda(d x)=q(x) d x$ while $\left\{\pi_{j}: j \in J\right\}$ is an arbitrary but finite set of linear or central projections $\pi_{j}: R^{n} \rightarrow$ $Y_{j}$, see [1], [4], [5]; $\left(\operatorname{dim} Y_{j} \leq n-1\right)$. This includes the above classical case.

An important class of sets of uniqueness are the so-called additive sets, first introduced for the classical case by Fishburn et al. [1], [2].

Let $F(S)$ denote the class of all $f: X \rightarrow R$ of the special form

$$
f(x)=\sum_{j \in J} f_{j}\left(\pi_{j} x\right)
$$

with the $f_{j}$ as functions $f_{j}: Y_{j} \rightarrow R(j \in J)$ satisfying the integrability 
condition (2.10). The importance of this class is due to the fact that

$$
\int_{X} f d \mu=\int_{X} f d \mu_{s}=\int_{S} f d \lambda, \quad \text { for any } f \in F(S),
$$

and any measure $\mu$ on $X$ having the same marginals as $\mu_{s}$ (relative to the $\left.\pi_{j} ; j \in J\right)$. A subset $S$ of $X$ with $\lambda(S)<\infty$, is said to be additive if it admits a representation of the form

$$
S=\left\{x \in X: f(x)=\sum_{j \in J} f_{j}\left(\pi_{j} x\right) \geq 0\right\},
$$

with $f$ as a suitable function $f \in F(S)$ as in (1.1). One easily shows that each additive set $S$ in $X$ is a (strong) set of uniqueness, see Lemma 2.14.

An easy illustration, relative to the classical case, would be any product set $S=A_{1} \times \cdots \times A_{n}$ in $R^{n}$ with $m_{1}\left(A_{j}\right)<\infty$. For, then $S$ is of the form (1.3) with $f_{j}(\xi)=+1$ or $-n$, depending on whether $\xi \in A_{j}$ or $\xi \notin A_{j}$, respectively, $(\xi \in R)$. Or choose $S$ as an ellipsoid in $R^{n}$ having its axes parallel to the coordinate axes. On the other hand, if $S$ is an ellipsoid $S=\left\{x \in R^{n}: \sum_{i, j} a_{i j} x_{i} x_{j} \leq 1\right\}$ with $a_{1 k} \neq 0$ for some $k \geq 2$ then $S$ is not a set of uniqueness, in fact, it has the same projections as the ellipsoid $T$ obtained by replacing all $a_{1 j}$ by $-a_{1 j}(j \geq 2)$.

Naturally, one wonders whether conversely all sets of uniqueness might be additive. This was shown to be true for the classical case with $n=2$ independently by Fishburn, Lagarias, Reeds and Shepp [1] and by Kuba and Volcic [7], [9], each making an essential use of the results due to Lorentz [8]. And for a while it was conjectured, for the classical case, that the converse is always true.

One goal of the present paper is to show that this converse is false. In $\S 3$, for the classical case with $n \geq 3$, we construct a large class of sets $S$ of uniqueness which are definitely not additive. One example with $n=3$ (see 3.22) has $S$ as a subset of $R^{3}$ equal to the union of a tetrahedron, a prism, and two rectangular blocks parallel to the axes.

Our construction is based on a new generalized notion of additivity. Ordinary additive sets are said to be additive of degree 1. A (stepwise) additive set $S$ of degree $\leq 2$ has the form $S=\{x \in X$ : either $f(x)>0$ or $f(x)=0 ; g(x) \geq 0\}$ with $f, g$ as suitably chosen functions in the class $F(S)$. In an analogous way, one defines the notion of an additive set $S$ of degree $\leq m$, see Definition 3.1. It is shown that each such generalized additive set $S$ is a (strong) set of 
uniqueness, see Theorem 3.1. Whenever the latter set $S$ can be shown to be non-additive, one has an example of a set of uniqueness which is not an additive set. In $\S 3$, this program is worked out in detail for the classical case with $n \geq 3$, starting with a suitably chosen additive set of degree $\leq 2$. There are still many open problems here.

Let us now restrict ourselves to the case where the set $X$ is $f$ nite, though we allow for the possibility of infinitely many projections $\pi_{j}: X \rightarrow Y_{j}(j \in J)$. In this situation, generalized additivity is the same as ordinary additivity, see Lemma 3.8. Moreover, a subset $S$ of $X$ is a (strong) set of uniqueness if and only if it is additive, see Theorem 4.50. For the special case that $X=[1,2, \ldots, N]^{n}$ with coordinate projections $x \rightarrow x_{j}(j=1, \ldots, n)$, the latter result is due to Fishburn, Lagarias, Reeds and Shepp [2]. The analogous result for weak sets of uniqueness is false, see [2] for a counterexample.

The proof of Theorem 4.50 is largely based on a new set of necessary and sufficient conditions in order that a solution $x^{0}$ of an arbitrary finite system of linear inequalities of type $\sum_{j} a_{i j} x_{j} \geq b_{i}(i=$ $1, \ldots, m) ; \alpha_{j} \leq x_{j} \leq \beta_{j}(j=1, \ldots, n)$ be the only such solution. One necessary condition is that $x^{0}$ be additive in a certain sense. See Theorem 4.15 for further details.

2. Sets of uniqueness. Let $X$ be a measurable space and $J$ an arbitrary index set. For each $j \in J$, let $Y_{j}$ be a measurable space and $\pi_{j}: X \rightarrow Y_{j}$ a measurable map. The collection $\pi=\left\{\pi_{j} ; j \in J\right\}$ of projections is kept fixed from now on. If $\mu$ is any measure on $X$ then its so-called $\pi_{j}$-marginal (or $\pi_{j}$-projection) is the measure $\pi_{j} \mu$ on $Y_{j}$ defined by $\left(\pi_{j} \mu\right)(B)=\mu\left(\pi_{j}^{-1} B\right)$. It follows that, for each measurable function $g_{j}: Y_{j} \rightarrow R$,

$$
\int_{X} g_{j}\left(\pi_{j} x\right) \mu(d x)=\int_{Y_{j}} g_{j}(y)\left(\pi_{j} \mu\right)(d y)
$$

provided $g_{j}$ is either nonnegative or $\left(\pi_{j} \mu\right)$-integrable. In particular, this integral is fully determined by the marginal $\pi_{j} \mu$ of $\mu$. Thus, if another measure $\nu$ on $X$ has the same marginals as $\mu$, that is, $\pi_{j} \nu=\pi_{j} \mu(j \in J)$, then

$$
\begin{aligned}
& \int_{X} g(x) \nu(d x)=\int_{X} g(x) \mu(d x) \\
& \text { if } g: X \rightarrow R \text { is of the form } g(x)=\sum_{j \in J} g_{j}\left(\pi_{j} x\right) .
\end{aligned}
$$

Here, the $g_{j}: Y_{j} \rightarrow R$ are assumed to be measurable such that $\sum_{j \in J} \int_{s}\left|g_{j}\left(\pi_{j} x\right)\right| \mu(d x)<\infty$. 
2.2. In the sequel, $\lambda$ is a fixed finite or $\sigma$-finite measure on $X$. We shall identify functions on $X$ (or subsets of $X$ ) which are equal a.e. $[\lambda]$. Define $M_{0}$ as the collection of measurable functions

$$
M_{0}=\left\{\phi: X \rightarrow R ; 0 \leq \phi(x) \leq 1(x \in X) ; \int_{X} \phi d \lambda<\infty\right\} ;
$$

(the latter condition is automatic when $\lambda$ is finite). Further $M$ will denote the associated class of (finite) measures $d \mu_{\phi}=\phi d \lambda$ on $X$ having density $\phi \in M_{0}$. If $\phi=1_{s}$ is the indicator function of a subset $S$ of $X$ then $\mu_{\phi}$ is also denoted as $\mu_{s}$; thus, $\mu_{s}(d x)=1_{s}(x) \lambda(d x)$. In the sequel, $S$ always denotes a measurable subset of $X$ satisfying $\lambda(S)<\infty$. Hence, $1_{s} \in M_{0}$, equivalently, $\mu_{s} \in M$.

For each $\mu \in M$, let

$$
M(\mu)=\left\{\nu \in M: \pi_{j} \mu=\pi_{j} \nu \text { for all } j \in J\right\},
$$

be the collection of all $\nu \in M$ having exactly the same marginals as $\mu$. Similarly for $\phi \in M_{0}$, let $M_{0}(\phi)$ be the set of all $\psi \in M_{0}$ such that $\mu_{\psi}$ has the same marginals as $\mu_{\phi}$; that is, $\mu_{\psi} \in M\left(\mu_{\phi}\right)$. In particular, $\mu \in M(\mu)$ and $\phi \in M_{0}(\phi)$.

2.4. Definition. We will call a function $\phi: X \rightarrow R$ a uniqueness function if $\phi \in M_{0}$ and, moreover, $M_{0}(\phi)=\{\phi\}$. Equivalently, $M\left(\mu_{\phi}\right)=\left\{\mu_{\phi}\right\}$.

We will say that a set $S$ is a weak set of uniqueness if there exists no measurable subset $T$ of $X$ distinct from $S$, such that $d \mu_{T}=1_{T} d \lambda$ has the same marginals as $d \mu_{s}=1_{s} d \lambda$. Equivalently, $M_{0}\left(1_{s}\right)$ contains no indicator function $1_{T}$ different from $1_{S}$. But we do not rule out the possibility that $\psi \in M_{0}\left(1_{s}\right)$ for some non-indicator function $0 \leq \psi \leq 1$ distinct from $1_{s}$. If also that possibility is ruled out then we will say that $S$ is a set of uniqueness. In other words, a subset $S$ of $X$ is a (strong) set of uniqueness if its indicator function $1_{s}$ is a uniqueness function, equivalently, if $M_{0}\left(1_{s}\right)=\left\{1_{s}\right\}$.

All these notions are relative to the given choice of the measure $\lambda$ on $X$ and maps $\pi_{j}: X \rightarrow Y_{j}(j \in J)$ and the standing assumption that $\lambda(S)<\infty$.

2.5. Since we identify sets $S, T$ or functions $\phi, \psi$ differing only on a $\lambda$-null set, one could identify a measurable set $S$ or a function $\phi \in M_{0}$ with the corresponding measure $d \mu_{s}=1_{s} d \lambda$ or $\phi d \lambda$, respectively. For instance, one could speak of the marginals of $S$ or 
$\phi$. These marginals of $S$ are analogous to the X-ray pictures from different directions of an object in $R^{3}$.

The set $S$ is a set of uniqueness set precisely when there is no $\psi \in M_{0}$ different from $1_{s}$, such that $d \mu_{\psi}=\psi d \lambda$ has exactly the same marginals as $d \mu_{s}=1_{s} d \lambda$. It implies that $S$ is also a weak set of uniqueness, meaning that there exists no other subset $T$ of $X$ can have the same marginals as $S$.

In many applications, the two notions are equivalent, in other words, any weak set $S$ of uniqueness is also a (strong) set of uniqueness. This equivalence holds, for instance, when $X, \lambda$ and $\pi_{j}(j \in J)$ are as in the following Example 2.76 and moreover, $\lambda$ is finite, see [1] and [4]. However, it fails in Example 2.7, see [2].

2.6. EXAMPLE. The following system $\left\{X, \lambda, \pi_{j}(j \in J)\right\}$ will be referred to as the classical case. Here, $m_{n}(d x)=d x$ denotes $n$ dimensional Lebesgue measure on $R^{n}$, while 'measurable' is the same as $m_{n}$-measurable. Namely, choose $X$ as a measurable subset of $R^{n}$ and $\lambda$ as an (absolutely continuous) measure $\lambda(d x)=q(x) d x$. Often one will choose $\lambda$ as the restriction of $m_{n}$ to $X$; it would be finite when $m_{n}(X)<\infty$. In addition, choose $J=\{1, \ldots, n\}$ and $\pi_{j}: X \rightarrow R$ as the projection which maps $x=\left(x_{1}, \ldots, x_{n}\right)$ to its $j$ th coordinate $x_{j}(j=1, \cdots, n)$.

We are interested in finite measures $\mu$ on $X$ of the form $d \mu=$ $\phi d \lambda=\phi(x) q(x) d x ; 0 \leq \phi \leq 1$, (often $\phi=1_{s}$ ). In the present example, the $\pi_{j} \mu(j=1, \cdots, n)$ are precisely the one-dimensional marginals of $\mu$. Clearly, $\pi_{j} \mu$ is an absolutely continuous measure on $R$ with density function

$$
\begin{aligned}
h_{j}(\xi)=\int_{X} \phi(x) q(x) d x_{1} \cdots d x_{j-1} d x_{j+1} \cdots d x_{n}, \\
\quad\left(x_{j}=\xi \in R ; j=1, \ldots, n\right) .
\end{aligned}
$$

Moreover, $\phi$ is a uniqueness function precisely when no other function $0 \leq \psi \leq 1$ leads to precisely the same marginal densities $h_{j}(j=$ $1, \cdots, n)$.

2.7. EXAmple. Let $X=\{1,2, \ldots, a\}^{n}$ and choose $J$ and the $\pi_{j}$ as in Example 2.6. Let $\lambda(x)=\lambda(\{x\})$. Presently, the $j$ th marginal $\pi_{j} \mu$ of a measure $d \mu=\phi d x$ on $X$ is the measure on $\{1, \ldots, a\}$ 
with discrete density function

$$
\begin{aligned}
h_{j}(\xi) & =\left(\pi_{j} \mu\right)(\{\xi\}) \\
& =\sum\left\{\phi\left(x_{1}, \ldots, x_{n}\right) \lambda\left(x_{1}, \ldots, x_{n}\right): x \in X ; x_{j}=\xi\right\}, \\
& \{\xi=1, \ldots, a ; j=1, \ldots, n\} .
\end{aligned}
$$

2.8. Definition. Given a set $S$ with $\lambda(S)<\infty$, let $F(S)$ denote the linear set consisting of all functions $f: X \rightarrow R$ having the special form

$$
f(x)=\sum_{j \in J} f_{j}\left(\pi_{j} x\right),
$$

with the $f_{j}: Y_{j} \rightarrow R(j \in J)$ as measurable functions such that

$$
\sum_{j \in J} \int_{S}\left|f_{j}\left(\pi_{j} x\right)\right| \lambda(d x)<\infty .
$$

Note from (2.1) that each $f \in F(S)$ is integrable relative to each $\mu \in M\left(\mu_{s}\right)$ while, moreover, the corresponding integral $\int f d \mu$ is fully determined by the marginals $\pi_{j} \mu_{s}(j \in J)$ of $\mu_{s}$.

Let further $F$ be the class of all $f: X \rightarrow R$ of the form (2.9) and satisfying

$$
\sum_{j \in J} \int_{X}\left|f_{j}\left(\pi_{j} x\right)\right| \lambda(d x)<\infty .
$$

The latter condition requires in particular that $f_{j}$ is $\left(\pi_{j} \lambda\right)$-integrable, as happens when $\lambda$ is finite and $f_{j}$ is bounded. Clearly, $F(S) \supset F$ for any $S$. Further each $f \in F$ is integrable relative to each $\mu \in M$. Of special interest is the case that all but finitely many $f_{j}(j \in J)$ are identically zero.

2.11. Definition. A subset $S$ of $X$ is said to be additive if $S$ is measurable, with $\lambda(S)<\infty$, and, moreover, $S$ is of the form

$$
S=S_{f}=\{x \in X: f(x) \geq 0\}, \quad \text { for some } f \in F(S) .
$$

Note that $1_{s}$ and $S$ attain the suprema

$$
\begin{aligned}
\sup & \left\{\int f \phi d \lambda: 0 \leq \phi \leq 1\right\} \\
& =\int f_{+} d \lambda=\sup _{T}\left\{\int_{T} f d \lambda\right\}=\int_{S} f d \lambda,
\end{aligned}
$$

(where $f_{+}=\max (0, f)$ ). For instance, relative to Example (2.6), the closed unit ball $S$ in $R^{n}$ is an additive set, as is seen by choosing 
$f(x)=1-\sum_{j} x_{j}^{2}=1-\|x\|^{2}$. In fact, $S$ maximizes $\int_{T}\left(1-\|x\|^{2}\right) d x ;$ therefore, $S$ minimizes $\int_{T}\|x\|^{2} d x$ among subsets $T$ of $R^{n}$ volume $\int_{T} d x=\int_{S} d x$.

The following result is a straightforward generalization of a result due to Fishburn et al. [2].

2.14. LeMMA. Each additive set $S$ is also a (strong) set of uniqueness.

2.15. Proof. Let $S$ be of the form (2.12), with $\lambda(S)<\infty$, and suppose that $d \nu=\psi d \lambda$ with $0 \leq \psi \leq 1$ has the same marginals as $d \mu=1_{s} d \lambda$ (thus, $\nu(X)=\mu(X)=\lambda(S)<\infty$ and $\left.\psi \in M_{0}\right)$. We must show that $\nu=\mu$, that is, $\psi(x)=1_{s}(x)$ a.e. [ $\left.\lambda\right]$. It follows from (2.1) that $\int f d \nu=\int f d \mu$, that is,

$$
\int_{X} f(x)\left(1_{s}(x)-\psi(x)\right) \lambda(d x)=0 .
$$

From $0 \leq \psi \leq 1$ and the definition of $S$, the latter integrand is nonnegative and thus zero a.e. [ $\lambda]$. This shows that $\psi(x)=1_{s}(x)$ a.e. [ $\lambda$ ] outside $D=\{x \in X: f(x)=0\}$. Here, $S \supset D$ thus $\lambda(D)<\infty$. Moreover,

$$
\begin{aligned}
\int_{D}(1-\psi(x)) \lambda(d x) & =\int_{D}\left(1_{S}(x)-\psi(x)\right) \lambda(d x) \\
& =\int_{X}\left(1_{S}(x)-\psi(x)\right) \lambda(d x)=0,
\end{aligned}
$$

the latter since $\int d \mu=\int d \mu=\lambda(S)<\infty$. Here, $1-\psi(x) \geq 0$; hence $\psi(x)=1=1_{S}(x)$ a.e. on $D$.

2.16. Property (A). We will say that the system $\left\{X, \lambda ; \pi_{j}(j \in J)\right\}$ has property (A) when, conversely, each set $S$ of uniqueness in $X$ is necessarily additive.

2.17. In this direction, we can report the following results.

(i) Property (A) does hold in the classical case (Example 2.6) when $n=2$ and $X=R^{2}$ while $\lambda$ is two-dimensional Lebesgue measure. This result is essentially due to Lorentz [8]; see Fishburn et al. [1]; Kuba and Volcic [7], [9] for related results.

(ii) Property (A) will be shown to be false in the classical case (Example 2.6) when $n \geq 3$ even when $X=[0,2]^{n}$ and $\lambda$ is Lebesgue measure. See $\S 3$ for details. 
(iii) Property (A) does hold for the discrete case of Example 2.7, as was shown in [2].

(iv) More generally, property (A) holds whenever $X$ is finite (even when $J$ is infinite). This result is obtained in $\S 4$ as a corollary to a uniqueness result for systems of linear inequalities.

3. Generalized additivity. Here, we use the notations of $\S 2$. We always assume that $\lambda(S)<\infty$. Recall the linear classes $F(S)$ and $F$ of functions $f: X \rightarrow R$ defined in 2.8. Further, an additive set $S$ is a subset of $X$ such that $S=\{x \in X: f(x) \geq 0\}$ for some $f \in F(S)$. From Lemma 2.12, each additive set is a set of uniqueness.

3.1. Definition. Let $S$ be a subset of $X$ with $\lambda(S)<\infty$. We will way that $S$ is (stepwise) additive of degree $\leq 2$ if it is of the form

$$
S=\{x \in X: f(x)>0\} \cup\{x \in X: f(x)=0 ; g(x) \geq 0\},
$$

for some $f, g \in F(S)$. More generally, $S$ will be said to be (stepwise) additive of degree $\leq m$ (with $m$ as a positive integer), if it has the following structure relative to a suitably chosen $m$-tuple of functions $f^{(r)} \in F(S)(r=1, \ldots, m)$.

(i) An element $x \in X$ belongs to $S$ if and only if the first non-zero element (if any) in the sequence $f^{(1)}(x), \ldots, f^{(m)}(x)$ is positive.

(ii) In particular, $x \in S$ whenever $f^{(r)}(x)=0$ for all $r=1$, $\ldots, m$.

Equivalently, $S$ is said to be additive of degree $\leq m$ if it is of the form

$$
S=U_{1} \cup U_{2} \cup \cdots \cup U_{m} \cup Z_{m},
$$

for some choice of the functions $f^{(r)} \in F(S)(r=1, \ldots, m)$. Here,

$$
\begin{aligned}
& U_{r}=\left\{x \in X: f^{(k)}(x)=0 \text { for } 1 \leq k<r ; f^{(r)}(x)>0\right\} ; \\
& Z_{r}=\left\{x \in X: f^{(r)}(x)=0 \text { for } 1 \leq k \leq r\right\} .
\end{aligned}
$$

3.5. We will further use the notation

$$
Q_{r}=\left\{x \in X: f^{(k)}(x)=0 \text { for } 1 \leq k<r ; f^{(r)}(x) \neq 0\right\} .
$$

Note that $X$ is the disjoint union of the sets $Q_{1}, \ldots, Q_{m}$ and $Z_{m}$. If $S$ is defined as in (3.3) and $x \in Q_{r}$, then $x \in S$ if and only if $f^{(r)}(x)>0(r=1,2, \ldots, m)$.

Additivity of degree $\leq m$ implies additivity of degree $\leq m+1$. This can be seen by choosing $f^{(m+1)}=f^{(m)}$, in which case $Q_{m+1}$ and thus $U_{m+1}$ is empty. We will say that $S$ is additive of degree $m$ if 
it is additive of degree $\leq m$ and, moreover, $m$ is minimal, that is, $S$ fails to be additive of any degree $\leq m^{\prime}$ for each $1 \leq m^{\prime}<m$. In particular, additivity of degree $\leq 1$, additivity of degree 1 and ordinary additivity (as in Definiton 2.11 ) are all equivalent.

3.7. Analogously, one defines the notion of a subset $S$ of $X$ to be additive of degree $\leq \infty$. This means that, for some infinite sequence $f^{(r)} \in F(S)(r=1,2, \ldots),$,$S happens to the union of all the$ associated sets $U_{r}(r \geq 1)$ as in (3.4), together with the set $Z_{\infty}=$ $\left\{x \in X: f^{(r)}(x)=0\right.$ for all $\left.r \geq 1\right\}$. If the latter set $S$ is not additive of any finite degree, we will say that $S$ is additive of infinite degree.

The following result shows that additivity of higher degree is no more general than ordinary additivity in the special case that $X$ is finite; (one may as well assume that $\lambda(X)<\infty$ ). Another proof for this discrete case would be as follows: (i) Theorem 3.11 below shows that an additive set of degree $\leq m$ is always a set of uniqueness. (ii) Theorem 4.50 below shows that each set of uniqueness is additive, as long as $X$ is finite.

3.8. LEMMA. If $X$ is finite then any subset $S$ of $X$ which is additive of degree $\leq m$, for some $1 \leq m \leq \infty$, is already additive (that is, additive of degree 1).

3.9. Proof. Let $1 \leq m \leq \infty$ and suppose $S$ is additive of degree $\leq m$ as in (3.3), (3.4), (or 3.7 when $m=+\infty$ ), where $f^{(r)} \in F(S)$. Functions $f^{(r)}$, such that $Q_{r}$ is empty, serve no purpose and may as well be dropped. In other words, one can assume without loss of generality that all sets $Q_{r}$ are non-empty $(1 \leq r \leq m)$. But $X$ is finite and the $Q_{r}$ are disjoint, hence, $m$ can be assumed to be finite. Let

$$
\begin{aligned}
& a=\inf _{x, r}\left\{\left|f^{(r)}(x)\right|: f^{(r)}(x) \neq 0\right\} ; \\
& b=\sup _{x, r}\left\{\left|f^{(r)}(x)\right|\right\} ; \quad \text { thus } 0<a \leq b<\infty,
\end{aligned}
$$

(where, $x \in X ; 1 \leq r \leq m)$. Choose further $0<\rho<a /(a+b)$ thus $\rho /(1-\rho)<a / b$, and consider

$$
f=f^{(1)}+\rho f^{(2)}+\rho^{2} f^{(3)}+\cdots+\rho^{r-1} f^{(r)} \text {, hence, } f \in F(S) .
$$

It suffices to show that $S$ coincides with the additive set

$$
T=\{x \in X: f(x) \geq 0\} .
$$


If $x_{0} \in Z_{m}$, that is, $f^{(k)}\left(x_{0}\right)=0$ for $1 \leq k \leq m$ then clearly $x_{0} \in S \cap T$. Next, let $x_{0} \notin Z_{m}$ and define $r=r\left(x_{0}\right)$ by $1 \leq r \leq$ $m ; f^{(k)}\left(x_{0}\right)=0$ for $1 \leq k<r$ and $f^{(r)}\left(x_{0}\right) \neq 0$. From (3.3), one has $x_{0} \in S$ or $x_{0} \in S^{c}$ depending on whether $f^{(r)}\left(x_{0}\right)>0$ or $f^{(r)}\left(x_{0}\right)<0$, respectively. It suffices to show that in these cases one has $f\left(x_{0}\right)>0$ or $f\left(x_{0}\right)<0$, respectively (to the effect that $x_{0} \in T$ or $x_{0} \notin T$, respectively). And that property follows immediately from

$$
\left|\sum_{r<k \leq m} \rho^{k-1} f^{(k)}\left(x_{0}\right)\right| \leq b \rho^{r} /(1-\rho)<a \rho^{r-1} \leq\left|\rho^{r-1} f^{(r)}\left(x_{0}\right)\right| .
$$

3.10. Let us return to the general case as in $\S 2$. For the classicial case described in Example 2.6 (with $n \geq 3$ ), Theorem 3.11 will enable us to construct sets of uniqueness which are not additive.

3.11. THEOREM. Let $S$ be a subset of $X$ which is additive of degree $\leq m$, for some $1 \leq m \leq \infty$. Then $S$ is a (strong) set of uniqueness.

3.12. Proof. Let $S$ be of the form (3.3) (or as in 3.7 when $m=$ $+\infty), \lambda(S)<\infty$. Let $0 \leq \psi \leq 1$ be such that the measure $d \nu=\psi d \lambda$ has the same marginals as $d \mu=1_{S} d \lambda$. In particular, from (2.1) and $f^{(r)} \in F(S)$, one has $\int f^{(r)} d \nu=\int f^{(r)} d \mu$, that is,

$$
\int f^{(r)}(x)\left(1_{S}(x)-\psi(x)\right) \lambda(d x)=0, \quad \text { for all } 1 \leq r \leq m .
$$

One must show that $\nu=\mu$, that is $\psi(x)=1_{S}(x)$ a.e. $[\lambda]$.

Let $Z_{r}$ be as in (3.4) (as in 3.7 when $r=\infty$ ), $Z_{0}=X$, and let $Q_{r}$ be as in (3.6). In particular, $Z_{0}=X$ is the disjoint union of the $m+1$ sets $Q_{r}$ and $Z_{m}(1 \leq r \leq m ; r<\infty)$. We first prove, by an induction on $r$, that $\psi(x)=1_{S}(x)$ a.e. [ $\lambda$ ] on $Q_{r}(r=1, \ldots, m ; r<\infty)$. Let $1 \leq r \leq m ; r$ finite, and suppose we already know that $\psi(x)=1_{S}(x)$ a.e. [ $[\lambda]$ or $Q_{k}$, for $1 \leq k<r$ and, thus, on the full complement $Q_{1} \cup \cdots \cup Q_{r-1}=X / Z_{r-1}$ of $Z_{r-1}$. Hence, (3.13) yields that

$$
\int_{Z_{r-1}} f^{(r)}(x)\left(1_{S}(x)-\psi(x)\right) \lambda(d x)=0 .
$$

Here, $Z_{r-1}=Q_{r} \cup Z_{r}$ with $Q_{r}$ and $Z_{r}$ disjoint. If $x \in Z_{r}$ then $f^{(r)}(x)=0$. On the other hand, if $x \in Q_{r}$ (thus $f^{(r)}(x) \neq 0$ ) we have from (3.3) and (3.4) that $x$ belongs to $S$ if and only if $f^{(r)}(x)>0$. Therefore, the integrand in (3.14) (where $0 \leq \psi \leq 1$ ) is nonnegative and thus equal to zero a.e. [ $\lambda]$ on $Z_{r-1}$ and thus on $Q_{r}$. Hence, $\psi(x)=1_{S}(x)$ a.e. [ $\left.\lambda\right]$ on $Q_{r}$ which completes our proof by induction. 
We now know that $\psi(x)=1_{S}(x)$ a.e. [ $\left.\lambda\right]$ on the full complement $Q_{1} \cup \cdots \cup Q_{m}=X / Z_{m}$. Further, $\nu(x)=\int \psi d \lambda=\int 1_{S} d \lambda<\infty, \quad$ thus $\int_{Z_{m}}\left(1_{S}(x)-\psi(x)\right) \lambda(d x)=0$.

But $S \supset Z_{m}$, thus, the latter integrand is nonnegative, showing that $\psi(x)=1_{S}$ a.e. [ $\left.\lambda\right]$ on $Z_{m}$. This completes the proof that $\psi(x)=1_{S}$ a.e. [ $[\lambda]$ on $X$.

3.15. REMARK. Theorem 3.11 and its proof even generalizes to sets $S$ which are additive of degree $\leq \alpha$, with $\alpha$ as any countable ordinal number. Here, an additive set $S$ of degree $\leq \alpha$ would be a subset $S$ with $\lambda(S)<\infty$, which is determined in the following manner by a suitable family $\left\{f^{(r)} ; r \leq \alpha\right\}$ in $F(S)$, with $r$ running through the ordinal numbers $r \leq \alpha$. If $x \in X$ is such that $f^{(r)}(x)=0$ for all $r \leq \alpha$ then $x \in S$. Otherwise, let $r=r(x)$ be the smallest ordinal number with $f^{(r)}(x) \neq 0$ and assign $x$ to $S$ or $S^{c}$, respectively, depending on whether $f^{(r)}(x)>0$ or $f^{(r)}(x)<0$, respectively. If $\alpha$ is minimal (given $S$ ) one would say that $S$ is additive of degree $\alpha$. It would be interesting to know (for instance, in the classical case 2.6) for what ordinal numbers $\alpha$ there does exist a subset $S$ of $X$, which is additive of degree $\alpha$. And also what systems $\left\{X, \lambda, \pi_{j}(j \in J)\right\}$ have the following Property (B): To every set $S$ of uniqueness in $X$ there corresponds a countable ordinal number $\alpha$, such that $S$ is additive of degree $\leq \alpha$. This property is of a similar type as Property (A), which was discussed in 2.16 and 2.17 .

The proof of Theorem 3.11 also yields that $T=U_{1} \cup U_{2} \cup \cdots \cup U_{m}$ is a set of uniqueness. However, this is nothing new, in fact, $T$ is additive of degree $\leq m+1$, as is seen by introducing $f^{(m+1)} \equiv-1$.

3.16. A non-additive set of uniqueness. From now on, in this section, we will restrict ourselves to the classical case as in 2.6. We will take $X$ as a bounded subset of $R^{n}$ supplied with Lebesgue measure. Further the $\pi_{j} \mu(j=1, \ldots, n)$ are the one-dimensional marginals of $d \mu=$ $\phi d \lambda$, with $0 \leq \phi \leq 1$. Assuming that $n \geq 3$ (as is necessary by 2.17), we would like to have an example of a subset of $S$ of $R^{n}$ which is additive of exact degree 2. This means that:

(i) $S$ is additive of degree $\leq 2$, (as in (3.2)). Thus, from Theorem $3.11, S$ is also a set of uniqueness.

(ii) $S$ is not additive. 
3.17. Construction. Let $m$ be a fixed integer, such that $2 \leq m \leq$ $n-1$ (as is only possible when $n \geq 3$ ). We will choose $X$ as the block

$$
\begin{aligned}
X & =[0,2)^{m} \times[0,1)^{n-m} \\
& =\left\{x \in R^{n}: 0 \leq x_{j}<2 \text { for } j \leq m ; 0 \leq x_{j}<1 \text { for } j>m\right\} .
\end{aligned}
$$

The reference measure $\lambda$ is chosen as Lebesgue measure $m_{n}$ restricted to $X$. One has $F(S) \supset F$ with $F$ as the class of all $f: X \rightarrow R$ of the form $f(x)=\sum_{j} f_{j}\left(x_{j}\right)$ with $f_{j}$ integrable on $[0,2)$ or $[0,1)$.

It will be convenient to regard the block $X$ as the disjoint union of the $2^{m}$ unit cubes $X_{\delta}$ defined by

$$
X_{\delta}=\left\{x \in X: \delta_{j} \leq x_{j}<\delta_{j}+1, \text { for } j=1, \ldots, m\right\}
$$

$\left(\delta_{j} \in\{0,1\}\right)$. Here, $\delta$ runs through all the $2^{m}$ sequences $\delta=$ $\left(\delta_{1}, \ldots, \delta_{m}\right)$ with $\delta_{j} \in\{0,1\}$. The choice $\delta=0$ leads to the special unit cube $X_{0}=\left\{x \in R^{n}: 0 \leq x_{j}<1\right.$, for $\left.j=1, \ldots, n\right\}$. Let $S_{\delta}=S \cap S_{\delta}$ denote the part of $S$ in $S_{\delta}$.

The subset $S$ of $X$ will be defined as in (3.2), with $f, g \in F$; hence $S$ is additive of degree $\leq 2$. Here, $f$ and $g$ are chosen to be of the special form

$$
f(x)=\sum_{j=1}^{m} f_{j}\left(x_{j}\right) ; \quad g(x)=\sum_{j=1}^{n} g_{j}\left(x_{j}\right)=\sum_{j=1}^{n} x_{j}-n+1 .
$$

Thus, $f_{j} \equiv 0$ for $m<j \leq n$. Further

$$
\begin{aligned}
& f_{j}\left(x_{j}\right)=0 \quad \text { if } 0 \leq x_{j}<1 \\
& f_{j}\left(x_{j}\right)=\frac{1}{m}-\left(x_{j}-1\right) \quad \text { if } 1 \leq x_{j}<2, \quad(j=1, \cdots, m)
\end{aligned}
$$

(much more general functions $f$ and $g$ would work equally well, see Remark 3.32 below). Observe that $f(x)=0$ for all $x \in X_{0}$. Hence, from (3.2),

$$
\begin{aligned}
S_{0} & =S \cap X_{0}=\left\{x \in X_{0}: g(x) \geq 0\right\} \\
& =\left\{x \in X_{0}: x_{1}+\cdots+x_{n} \geq n-1\right\} .
\end{aligned}
$$

Clearly, $S_{0}$ is a simplex (pyramid) in $R^{n}$ having the points $(0,1, \ldots$, $1) ; \cdots,(1, \ldots, 1,0)$ and $(1, \ldots, 1)$ as its $n+1$ vertices.

Ignoring the set $\left\{x \in S / X_{0} ; f(x)=0\right\}$, which has $m_{n}$-measure zero, the remaining part of $S$ is of the form

$$
S / X_{0}=\{x \in X: f(x)>0\}
$$


and thus independent of the function $g$. Let $\delta \neq 0$ where $\delta=$ $\left(\delta_{1}, \ldots, \delta_{m}\right)\left(\delta_{j} \in\{0,1\}\right)$. It is immediate, from the definition of $X_{\delta}$ and (3.19), that $S_{\delta}=S \cap X_{\delta}$ consists of all $x \in R^{n}$ satisfying

(i) $0 \leq x_{j}<1$ when $j>m$ or $\delta_{j}=0(j=1, \cdots, n)$;

(ii) If $\delta_{j}=1$ then $1 \leq x_{j}<2(j=1, \cdots, m)$ in such a way that

$$
\sum\left\{x_{j}-1: \quad \delta_{j}=1 ; 1 \leq j \leq m\right\}<d / m .
$$

Here, $d=|\delta|=\delta_{1}+\cdots+\delta_{m}$; hence, $1 \leq d \leq m$. Thus, $S_{\delta}$ is a prism $S_{\delta}=B_{\delta} \times[0,1)^{n-d}$ having as its base $B_{\delta}$ a simplex in $R^{d}$, with vertices $\left(y_{1}, \ldots, y_{d}\right)$ for which $y_{j}=1$, with at most one exception, in which case $y_{j}=1+d / m$. If $d=1$ this base is an interval and $S_{\delta}$ is a rectangular block. For instance, if $\eta=(1,0, \ldots, 0)$ then $S_{\eta}=[1,1+1 / m) \times[0,1)^{n-1}$. If $d=2$ then the base $B_{\delta}$ is a triangle.

3.23. Consider the special case $n=3$ where $m=2$. Then our set $S$ of uniqueness in $R^{3}$ consists of: (i) The pyramid (tetrahedron) with vertices $(1,1,1) ;(0,1,1) ;(1,0,1) ;(1,1,0)$. (ii) The rectangular blocks $[1,3 / 2) \times[0,1) \times[0,1)$ and $[0,1) \times[1,3 / 2) \times[0,1)$. (iii) The prism $B \times[0,1)$ with $B$ as the triangle $B=\left\{\left(x_{1}, x_{2}\right): x_{1} \geq\right.$ $\left.1 ; x_{2} \geq 1 ; x_{1}+x_{2}<3\right\}$.

3.23. THEOREM. The set $S$ of uniqueness as constructed above is not additive.

3.24. Proof. On the contrary, suppose there exist measurable functions $h_{1}, \ldots, h_{m}$ on $[0,2)$; further measurable functions $h_{m+1}, \ldots$, $h_{n}$ on $[0,1)$ and a subset $N$ of $X$, with $m_{n}(N)=0$, such that if $x=\left(x_{1}, \ldots, x_{n}\right) \in X / N$ then

$$
\sum_{j} h_{j}\left(x_{j}\right) \geq 0 \text { if and only if } x \in S .
$$

In the sequel, indices $j, r$ and $s$ will run through $\{1, \ldots, n\}$; $\{1, \ldots, m\}$ and $\{m+1, \ldots, n\}$, respectively. We write $x=$ $\left(x_{1}, \ldots, x_{n}\right) \in R^{n}$ as $x=(y, z)$, where $y=\left(y_{1}, \ldots, y_{m}\right)=\left(x_{1}, \ldots\right.$, $\left.x_{m}\right)$ and $z=\left(z_{m+1}, \ldots, z_{n}\right)=\left(x_{m+1}, \ldots, x_{n}\right)$. Let further

$$
\begin{aligned}
H_{1}(y) & =\sum_{r} h_{r}\left(y_{r}\right) ; & H_{2}(z) & =\sum_{s} h_{s}\left(z_{s}\right) ; \\
L_{1}(y) & =\sum_{r} y_{r} ; & L_{2}(z) & =\sum_{s} z_{s} .
\end{aligned}
$$


Thus, (3.25) can be written as if $(y, z) \in X / N$ then

$$
H_{1}(y)+H_{2}(z) \geq 0 \text { if and only if }(y, z) \in S .
$$

In particular, (3.20) implies that if $(y, z) \in X_{0} / N$ then (3.27) $H_{1}(y)+H_{2}(z) \geq 0$ if and only if $L_{1}(y)+L_{2}(z) \geq n-1$.

Next, let us apply (3.26) for points $(y, z) \in X_{\zeta}$ where $\zeta=$ $(1, \ldots, 1)$. Note that $X_{\zeta}=[1,2)^{m} \times(0,1)^{n-m}=e+X_{0}$, where $e \in R^{n}$ has coordinates $e_{r}=1(r=1, \ldots, m)$ and $e_{s}=0(s=$ $m+1, \ldots, n)$. We see from (3.21) with $\delta=\zeta$ (thus $d=|\delta|=m$ ) that

$$
S \cap X_{\zeta}=\left\{x \in X_{\zeta}: \sum_{r}\left(x_{r}-1\right)<1\right\}=e+\left\{(y, z) \in X_{0}: L_{1}(y)<1\right\} .
$$

Hence, it follows from (3.27) that if $(y, z) \in X_{0} / N_{1}$ then

$$
K_{1}(y)+H_{2}(z) \geq 0 \text { if and only if } L_{1}(Y)<1 \text {. }
$$

Here,

$$
K(y)=\sum_{r} k_{r}\left(y_{r}\right) \quad \text { where } k_{r}(\xi)=h_{r}(1+\xi) \quad(r=1, \ldots, m)
$$

$\left(y \in[0,1)^{m} ; \xi \in[0,1)\right)$. Further, $N_{1}=\left\{x \in X_{0}: e+x \in N\right\}$ thus $m_{n}\left(N_{1}\right)=0$. Note that $\left\{(y, z) \in X_{0}: L_{1}(y)=0\right\}$ has $m_{n}$-measure zero and can be ignored.

We will show that (3.27), (3.28) together lead to a contradiction. In the sequel, $y$ ranges through $I^{m}$ and $z$ through $I^{n-m}$, where $I=[0,1)$. Put

$$
\alpha=\operatorname{essinf}\left\{H_{2}(z)\right\}: \quad \beta=\operatorname{ess} \sup \left\{H_{2}(z)\right\} ;
$$

thus, $\alpha \leq \beta$, (possibly $\alpha=-\infty$ or $\beta=+\infty$ ). We claim that $\alpha<\beta$.

Suppose not, equivalently, $\alpha$ is finite and $H_{2}(z)=\alpha$ a.e. on $I^{n-m}$. Since $m_{n}(N)=0$, there exists a subset $E$ of $I^{n-m}$ with $m_{n-m}(E)=$ 0 , such that, for each $z \in I^{n-m} / E$, one has $(y, z) \notin N$ for almost all $y \in I^{m}$. Note that the linear function $L_{1}(y)=\sum_{r} y_{r}$ on $I^{m}$ takes all values in $[0, m)$, while the linear function $L_{2}(z)=\sum_{s} z_{s}$ on $I^{n-m}$ takes all values in $[0, n-m)$. Clearly, there exist $z^{\prime}, z^{\prime \prime} \in$ $I^{n-m} / E$ such that $H_{2}\left(z^{\prime}\right)=H_{2}\left(z^{\prime \prime}\right)=\alpha$; further $L_{2}\left(z^{\prime}\right)<L_{2}\left(z^{\prime \prime}\right)$ and $n-m-1<L_{2}\left(z^{\prime \prime}\right)<n-m$. Next, there exists $y \in I^{m}$ such that

$$
\begin{aligned}
& \left(y, z^{\prime}\right) \notin N,\left(y, z^{\prime \prime}\right) \notin N \text { and } \\
& n-1-L_{2}\left(z^{\prime \prime}\right)<L_{1}(y)<n-1-L_{2}\left(z^{\prime}\right) ;
\end{aligned}
$$


(just choose $y$ such that $L_{1}(y)$ is slightly larger than the value $p=$ $n-1-L_{2}\left(z^{\prime \prime}\right)$, which satisfies $\left.m-1<p<m\right)$. Applying (3.27), we see that $\left(y, z^{\prime}\right) \notin N$ and $L_{1}(y)+L_{2}\left(z^{\prime}\right)<n-1$ imply $H_{1}(y)+\alpha<$ 0 . Similarly, $\left(y, z^{\prime \prime}\right) \notin N$ and $L_{1}(y)+L_{2}\left(z^{\prime \prime}\right)>n-1$ imply that $H_{1}(y)+\alpha \geq 0$ and we have a contradiction. This proves that $\alpha<\beta$.

Since $m_{n}\left(N_{1}\right)=0$ there exists a subset $D$ of $I^{m}$ of ( $m$-dimensional Lebesgue) measure zero, such that, for each $y \in D$, one has $(y, z) \notin$ $N_{1}$ for almost all $z \in I^{n-m}$. It follows from (3.28) and (3.29) that

\section{(3.30) if $y \in I^{m} / D$ and $L_{1}(y)<1$ then $K_{1}(Y)+\alpha \geq 0$; if $y \in I^{m} / D \quad$ and $\quad L_{1}(y) \geq 1$ then $K_{1}(y)+\beta \leq 0$.}

Here, $L_{1}(y)=\sum y_{r}$ takes all values in $[0, m)$, thus, $\alpha$ and $\beta$ are finite. Let $\Delta=\beta-\alpha$; thus $\Delta>0$. At least intuitively, (3.30) seems impossible. For, it would imply that, roughly speaking, $K_{1}(y)=$ $\sum_{r} k_{r}\left(y_{r}\right)$ makes a downward jump of size $\geq \Delta$ everywhere across the hyperplane $L_{1}(y)=\sum y_{r}=1$. Here, $r=1, \ldots, m$ where $m \geq 2$.

Let us write $y \in I^{m}$ as $y=(u, \nu)$, where $u=\left(u_{1}, \ldots, u_{m-1}\right)=$ $\left(y_{1}, \ldots, y_{m-1}\right) \in I^{m-1}$ and $v=y_{m} \in I=[0,1)$. Thus $L_{1}(y)=$ $L_{1}(u, v)=L_{0}(y)+v$ where $L_{0}(u)=u_{1}+\cdots+u_{m-1}$. Further $K_{1}(y)=$ $K_{1}(u, v)=K_{0}(u)+k(v)$, where $K_{0}(u)=k_{1}\left(u_{1}\right)+\cdots+k_{m-1}\left(u_{m-1}\right)$ and $k(v)=k_{m}(v)$.

Let $C$ be a subset of $I=[0,1)$ of full measure $m_{1}(C)=1$ such that $(u, v) \notin D$, for almost all $u \in I^{m-1}$, whenever $v \in C$. Let $v^{\prime}, v^{\prime \prime} \in C$ with $v^{\prime}<v^{\prime \prime}$ be fixed for the moment. There exists $u \in I^{m-1}$ such that $\left(u, v^{\prime}\right) \notin D ;\left(u, v^{\prime \prime}\right) \notin D$ and $1-v^{\prime \prime}<L_{0}(u)<$ $1-v^{\prime}$; (after all, $L_{0}(u)$ is a linear function on $I^{m-1}$ taking all values in $(0, m-1)$ and thus all values in $\left(1-v^{\prime \prime}, 1-v^{\prime}\right)$; it is mainly here that $m \geq 2$ is used). Since $\left(u, v^{\prime}\right) \notin D$ and $L_{1}\left(u, v^{\prime}\right)=L_{0}(u)+$ $v^{\prime}<1$, it follows from (3.30) that $K_{1}\left(u, v^{\prime}\right)=K_{0}(u)+k\left(v^{\prime}\right) \geq-\alpha$. Similarly, since $\left(u, v^{\prime \prime}\right) \notin D$ and $L_{0}(u)+v^{\prime \prime}>1$, one has that $K_{0}(u)+$ $k\left(v^{\prime \prime}\right) \leq-\beta$. Consequently, $k\left(v^{\prime \prime}\right)-k\left(v^{\prime}\right) \leq-\beta+\alpha=-\Delta<0$ whenever $v^{\prime}, v^{\prime \prime} \in C$ with $v^{\prime}<v^{\prime \prime}$. Choosing $v_{i} \in C$ with $v^{\prime}<$ $v_{1}<\cdots<v_{M-1}<v^{\prime \prime}$, it would follow that $k\left(v^{\prime \prime}\right)-k\left(v^{\prime}\right) \leq M \Delta$ for $M$ arbitrarily large and this is clearly impossible. This completes the proof of Theorem 3.23.

3.31. REMARK. Omitting the proof, we assert that (3.25) restricted to $X_{0}$ (which is the same as (3.27)) has only trivial solutions $h=$ $\left(h_{1}, \ldots, h_{n}\right)$, provided $n \geq 3$. More precisely, suppose that $h_{j}: I=$ $[0,1) \rightarrow R(j=1, \cdots, n)$ are measurable functions such that, for 
all $x \in I^{n} / N$, one has $\sum_{j} h_{j}\left(x_{j}\right) \geq 0$ if and only if $\sum_{j} x_{j} \geq n-1$. Here, the subset $N$ of $I^{n}$ satisfies $m_{n}(N)=0$. Then there exist constants $a>0$ and $b_{j}$ such that $h_{j}\left(x_{j}\right)=a x_{j}-b_{j}$ for almost all $x_{j}=I(j=1, \cdots, n)$. Naturally, $\sum_{j} b_{j}=(n-1) a$. The analogous result with $n=2$ is false. After all, for each choice of the strictly increasing function $\theta:[0,1] \rightarrow R, x_{1}+x_{2} \geq 1$ a.e. on $[0,1)^{2}$ is equivalent to $\theta\left(x_{1}\right)-\theta\left(1-x_{2}\right) \geq 0$ a.e. on $[0,1)^{2}$.

3.32. REMARK. The proof of Theorem 3.23 is quite robust and uses very few of the properties of the functions $f, g \in F$ defining $S$ as in (3.2). Let us maintain the structure of $X$ and also the assumptions that $2 \leq m \leq n-1$ and

$$
\begin{gathered}
f(x)=\sum_{j=1}^{m} f_{j}\left(x_{j}\right), \quad \text { with } f_{j}\left(x_{j}\right)=0 \text { if } 0 \leq x_{j}<1 ; \\
g(x)=\sum_{j=1}^{n} g_{j}\left(x_{j}\right) \quad(x \in X) .
\end{gathered}
$$

In particular, $f_{j} \equiv 0$ if $j>m$. One has $f(x)=0$ for all $x \in X_{0}=$ $[0,1)^{n}$, thus, $S_{0}=S \cap X_{0}=\left\{x \in X_{0}: g(x) \geq 0\right\}$ is determined by the restrictions $g_{j}:[0,1) \rightarrow R(j=1, \cdots, n)$.

Writing $x \in X_{0}$ as $x=(y, z)$ with $y \in[0,1)^{m}$ and $z \in[0,1)^{n-m}$, we want that the property $(y, z) \in S_{0}$ depends in an essential way on $z$. This in order that, as in the above proof, $\alpha$ and $\beta$ as in (3.29) will satisfy $\alpha<\beta$. It would be sufficient that

$\underset{z}{\operatorname{essinf}} \sum_{s} g_{s}\left(z_{s}\right)<-\sum_{r} g_{r}\left(y_{r}\right)<\underset{z}{\operatorname{ess} \sup } \sum_{s} g_{s}\left(z_{s}\right), \quad z \in[0,1)^{n-m}$ $(r-1, \ldots, m ; s=m+1, \ldots, n)$ holds for a set of points $y \in[0,1)^{m}$ of positive measure. For each such $y$, the set $\left\{(y, z): z \in[0,1)^{n-m}\right\}$ meets both $S$ and $S^{c}$ in a set of positive measure.

The above method of proof easily leads to a contradiction (from the assumption that $S$ is additive), provided that, in addition, the functions $f_{1}, \ldots, f_{m}$ are continuous on $(1,2)$ in such a way that

$$
\sum_{r=1}^{m-1} f_{r}\left(x_{r}\right)<-f\left(x_{m}\right)<-f\left(y_{m}\right)<\sum_{r=1}^{m-1} f_{r}\left(y_{r}\right),
$$

for at least one choice of the numbers $x_{r}, y_{r} \in(1,2)(r=1, \ldots, m)$. This is a rather weak assumption. We omit the details. 
4. The finite case. Here, we characterize systems of linear inequalities having a unique solution. Let $m$ and $n$ be fixed positive integers and $I=\{1, \ldots, m\} ; J=\{1, \ldots, n\}$. Unspecified indices $i$ and $j$ run through $I$ and $J$, respectively. Further $A=\left(a_{i j}\right) \in R^{m n}$ is a given $m \times n$ matrix and $b=\left(b_{1}, \ldots, b_{m}\right)^{T} \in R^{m}$ a given column vector. We will be interested in the system of inequalities $A x \geq b$, with $x=\left(x_{1}, \ldots, x_{n}\right)^{T}$. That is,

$$
\sum_{j \in J} a_{i j} x_{j} \geq b_{i}, \quad \text { for all } i \in I \text {. }
$$

In addition, we impose the conditions

$$
\alpha_{j} \leq x_{j} \leq \beta_{j}, \quad \text { for all } j \in J .
$$

Here, and from now on, the $\alpha_{j}$ and $\beta_{j}$ are given extended real numbers such that

$$
-\infty \leq \alpha_{j}<\beta_{j} \leq+\infty \quad(j \in J)
$$

(the case $\alpha_{j}=\beta_{j}$, where $x_{j}=\alpha_{j}$ is prescribed, can be eliminated by lowering $n$ ).

Specifically, we shall be interested in characterizing the situations where (4.1), (4.2) have a unique solution $x^{0}$. The special case where (4.2) is void (that is $\alpha_{j}=-\infty ; \beta_{j}=+\infty$, for all $j \in J$ ) leads to the following definition.

4.4. Definition. The matrix $A=\left(a_{i j}\right)$ is said to be a matrix of uniqueness if

$$
x \in R^{n} ; \quad A x \geq 0 \text { imply that } x=0 .
$$

Naturally, this would be equivalent to $A x \leq 0$ implying that $x=0$.

Since the matrix $A$ represents a map $R^{n} \rightarrow R^{m}$, one is not allowed to identify all empty matrices (having $m=0$ or $n=0$ ). In fact, (4.5) forces the convention that an empty matrix with $m=0$ and $n \geq 1$ is never a matrix of uniqueness. It will be convenient to regard an empty $m \times n$ matrix with $n=0$ columns as being a matrix of uniqueness. The following result is known.

4.6. LEMMA. In order that $A$ be a matrix of uniqueness it is necessary and sufficient that

(4.7) for each $z \in R^{n}$ there exists $y \in R^{m}$

$$
\text { such that } y \geq 0 \text { and } y A=z \text {. }
$$

Here, $y$ and $z$ are row vectors. 
4.8. Proof. Assume property (4.7) and let $x \in R^{n}$ satisfy $A x \geq 0$. Applying (4.7) with $z=-x^{T}$, there exists $y \in R^{m}$ with $y \geq 0$ and $y A=-x^{T}$. Hence,

$$
0 \leq y(A x)=(y A) x=-x^{T} x=-\|x\|^{2}, \quad \text { thus }, x=0 .
$$

Next, suppose property (4.7) is false. That is, some $z_{0} \in R^{n}$ is not contained in the convex cone $K=\{y A: y \geq 0\}$. It follows that $K$ is contained in some closed half space $H_{+}=\left\{z \in R^{n}: z c \geq 0\right\}$ with $c \in R^{n}$ as a non-zero column vector. Thus $y(A c) c \geq 0$, whenever $y \geq 0$; hence, $A c \geq 0$ contradicting (4.5).

4.9. Remark. Geometrically, Lemma 4.6 is very intuitive. For (4.5) requires that the finite collection of closed half spaces $\sum_{j} a_{i j} x_{j} \geq$ $0(i \in I)$ has intersection $\{0\}$. And property (4.7) says that the convex cone spanned by the associated normals (the rows of $A$ ) is all of $R^{n}$.

4.10. REMARK. The system of equalities $A x=0$ would be equivalent to $B x \geq 0$, where $B$ is the $2 m \times n$ matrix $B=\left(\begin{array}{c}A \\ -A\end{array}\right)$. Clearly, $B$ is a matrix of uniqueness if and only if $A$ has $\operatorname{rank} n$. Condition (4.7) applied to $B$ (instead of $A$ ) says that the rows of $A$ span all of $R^{n}$.

4.11. In the sequel, $x^{0}$ is a fixed solution of (4.1) and (4.2), while $I^{0}$ and $J^{0}$ denote the associated index sets

$$
I^{0}=\left\{i \in I: \sum_{j} a_{i j} x_{j}^{0}=b_{i}\right\}
$$

$$
J^{0}=\left\{j \in J: \alpha_{j}<x_{j}^{0}<\beta_{j}\right\} .
$$

Further $A^{0}$ denotes the (possibly empty) associated submatrix of $A$ defined by

$$
A^{0}=\left\{a_{i j} ; i \in I^{0} ; j \in J^{0}\right\}
$$

4.15. THEOREM. Let $x^{0}$ be a fixed solution of (4.1), (4.2) with associated $I^{0}, J^{0}$ and $A^{0}$ as above. Then in order that $x^{0}$ be the only solution of (4.1), (4.2), it is necessary and sufficient that $x^{0}$ satisfies the following two conditions. 
(i) There must exist a vector $f=\left(f_{1}, \ldots, f_{m}\right)$ of the form

$$
f_{j}=\sum_{i \in I} y_{i} a_{i j} ; \quad y_{i} \geq 0(i \in I) ; \quad y_{i}=0\left(i \notin I^{0}\right)
$$

and satisfying

$$
\begin{aligned}
& f_{j}<0 \quad \text { iff } x_{j}^{0}=\alpha_{j} ; \\
& f_{j}>0 \quad \text { iff } x_{j}^{0}=\beta_{j} ; \\
& f_{j}=0 \quad \text { iff } \alpha_{j}<x_{j}^{0}<\beta_{j} ; \quad \text { that is, iff } j \in J^{0} .
\end{aligned}
$$

(ii) The matrix $A^{0}$ defined by (4.14) is a matrix of uniqueness.

4.18. REMARKs. Note that the above uniqueness criterion depends on $b \in R^{m}$ only through the index set $I^{0}$ defined by (4.12). The vector $f$ in (ii) is often far from unique. Condition (ii) is automatically satisfied when $J^{0}$ is empty, that is, when $x_{j}^{0} \in\left\{\alpha_{j}, \beta_{j}\right\}$ for all $j \in J$.

The necessity of condition (ii) is easily seen. For, if (ii) were false then, from Definition 4.4 , there would exist a non-zero function $z: J \rightarrow R$ carried by $J^{0}$ (that is, $z_{j}=0$ if $x_{j}^{0}=\alpha_{j}$ or $x_{j}^{0}=\beta_{j}$ ) such that $\sum_{j} a_{i j} z_{j} \geq 0$ for all $i \in I^{0}$. Replacing $x^{0}$ by $x=x^{0}+p z$, with $p>0$ small, one obtains a different solution of (4.1), (4.2).

4.19. As a simple illustration, let $n=1$. Here, $x \in R$ is subject to $a_{i} x \geq b_{i}(i \in I)$ and $\alpha \leq x \leq \beta$ (where $\alpha<\beta$ ). A solution $x^{0}$ is unique if and only if one of the following happens. Here, $I^{0}=\{i \in$ $\left.I: a_{i} x^{0}=b_{i}\right\}$.

(i) One has $\alpha<x^{0}<\beta$, that is, $J^{0}=\{1\}$, and, moreover, there exist $r, s \in I^{0}$ with $a_{r}<0$ and $a_{s}>0$. Here, the above assertions hold with $y_{i}=0$, and thus $f=\sum_{i} y_{i} a_{i}=0$.

(ii) One has $\alpha=x^{0}<\beta$ (thus $J^{0}$ is empty) and, in addition, $a_{r}<0$ for some $r \in I^{0}$ (thus $a_{r} x^{0}=b_{r}$ ). Then choose $y_{r}=1$ and $y_{i}=0(i \neq r)$; hence, $f=\sum_{i} y_{i} a_{i}=a_{r}<0$.

(iii) One has $\alpha<x^{0}=\beta$ (thus $J^{0}$ is empty) and $a_{r}>0$ for some $r \in I^{0}$. Then choose $y_{r}=1$ and $y_{i}=(i \neq r)$ so that $f=a_{r}>0$.

4.20. Consider the case that $I^{0}$ is empty, that is, $A x^{0}>b$. Then $A x \geq b$ holds for all $x$ in a sufficiently small neighborhood of $x^{0}$ showing that $x^{0}$ cannot possibly be a unique solution of $(4.1),(4.2)$, which must mean that either (i) or (ii) is false. Indeed, $A^{0}$ is not a matrix of uniqueness unless possibly $J^{0}$ is empty, (see 4.4). But in the latter case, (i) is false. Namely, (i) with $I^{0}$ empty would imply that $f_{j}=0$ for all $j \in J$, thus, that $J^{0}=J \neq \varnothing$. 
4.21. Proof of Theorem 4.15. Sufficiency. Suppose $x^{0}$ satisfies not only (4.1), (4.2) but also (i) and (ii). Consider any solution $x$ of (4.1), (4.2). Multiplying (4.1) by $y_{i} \geq 0$ and summing, and using (4.16), one finds that $f(x):=\sum_{j} f_{j} x_{j} \geq \sum_{i} b_{i} y_{i}=f\left(x^{0}\right)$, and thus, $\sum_{j} f_{j}\left(x_{j}^{0}-x_{j}\right) \leq 0$. But, in view of (4.2) and (4.17), each term in the latter sum is nonnegative. Consequently, $x_{j}=x_{j}^{0}$ whenever $f_{j} \neq 0$, that is, whenever $j \notin J^{0}$. This already implies the uniqueness of $x^{0}$ when $J^{0}$ is empty.

Otherwise, it suffices to prove $z=0$ with $z$ as the restriction of $x-x^{0}$ to $J^{0}$. In fact, $\sum_{j} a_{i j} x_{j} \geq b_{i}=\sum_{j} a_{i j} x_{j}^{0}$, for each $i \in I^{0}$. We already know that $x_{j}=x_{j}^{0}$, for $j \notin J^{0}$, and hence $A^{0} z \geq 0$. But this implies $z=0$ since $A^{0}$ is a matrix of uniqueness.

4.22. Necessity. The necessity of condition (ii) was already established in 4.18. It remains to show that (4.16), (4.17) hold for some vector $f=\left(f_{1}, \ldots, f_{n}\right)$. The proof will be by induction with respect to $n=|J|$. The case $n=0$ is trivially true, while the case $n=1$ was verified in 4.19.

Decreasing $x_{j}, \alpha_{j}$ and $\beta_{j}$ by $x_{j}^{0}(j \in J)$ and decreasing $b_{i}$ by $\sum_{j} a_{i j} x_{j}^{0}(i \in I)$, one may without loss of generality assume that $x^{0}=0$. Thus, $x^{0}=0$ is the unique solution of (4.1), (4.2). Therefore

$$
\begin{aligned}
\alpha_{j} \leq 0 \leq \beta_{j}(j \in J) ; & J^{0}=\left\{j \in J: \alpha_{j}<0<\beta_{j}\right\} ; \\
b_{i} \leq 0(i \in I) ; & I^{0}=\left\{i \in I: b_{i}=0\right\} .
\end{aligned}
$$

We first observe that $x^{0}=0$ is also the unique solution of (4.2) together with the (homogeneous) system

$$
\sum_{j \in J} a_{i j} x_{j} \geq 0 \text { for all } i \in I^{0}
$$

(itself a subsystem of (4.1)). For, otherwise, there would exist $x \neq 0$ satisfying both (4.2) and (4.23). Since $b_{i}=0\left(i \in I^{0}\right)$ and $b_{i}<0(i \notin$ $\left.I^{0}\right), x^{\prime}=\rho x=(1-\rho) \cdot 0+\rho x$ would satisfy both (4.2) and the full system (4.1), as soon as $0<\rho<1$ is sufficiently small. But then $x^{0}=0$ would not be the unique solution of (4.1), (4.2).

The just established uniqueness property says that $K \cap Q=\{0\}$, where

$$
K=\left\{x \in R^{n}: \sum_{j \in J} a_{i j} x_{j} \geq 0, \text { for all } i \in I^{0}\right\}
$$

and

$$
Q=\left\{x \in R^{n}: \alpha_{j} \leq x_{j} \leq \beta_{j}, \text { for all } j \in J\right\}
$$


Here, $K$ is a closed and convex cone in $R^{n}$, possibly of the form $K=\{0\}$. Further $Q$ is a closed and convex subset of $R^{n}$ having a non-empty interior $\operatorname{int}(Q)$ (because $\alpha_{j}<\beta_{j}$ for all $j \in J$ ). One can separate $K$ and $Q$ by a hyperplane as soon as $K$ is disjoint from $\operatorname{int}(Q)$.

First, consider the case that $K$ is not disjoint from $\operatorname{int}(Q)$. Since $K \cap Q=\{0\}$, this is equivalent to $0 \in \operatorname{int}(Q)$, that is, $\alpha_{j}<0<\beta_{j}$ for all $j \in J$; equivalently, $J^{0}=J$. But then property (i) holds with $y_{i}=0$ for all $i \in I$; thus, $f_{j}=0$ for all $j \in J$.

It remains to consider the situation that $K \cap \operatorname{int}(Q)$ is empty. Then there exists a hyperplane $H=\left\{x \in R^{n}: \sum_{j} f_{j} x_{j}=0\right\}$ in $R^{n}$ with $f=\left(f_{1}, \ldots, f_{n}\right)$ non-zero such that $K$ is a subset of $H_{+}=\{x \in$ $\left.R^{n}: \sum_{j} f_{j} x_{j} \geq 0\right\}$ and $Q$ is a subset of $H_{-}=\left\{x \in R^{n}: \sum_{j} f_{j} x_{j} \leq 0\right\}$. From a classical result, see Gale [3], p. 44, the fact $H_{+} \supset K$ implies that there exist numbers $y_{i}(i \in I)$ as in (4.16).

Next, the fact $H_{-} \supset Q$ means that $\alpha_{j} \leq x_{j} \leq \beta_{j}(j \in J)$ implies $\sum_{j} f_{j} x_{j} \leq 0$. Recall that $\alpha_{j} \leq 0 \leq \beta_{j}(j \in J)$. Choosing all but one $x_{j}$ equal to zero, we have, for each $j \in J$, that $\alpha_{j} \leq x_{j} \leq \beta_{j}$ implies $f_{j} x_{j} \leq 0$. Equivalently,

$$
f_{j}<0 \text { implies } \alpha_{j}=0 ; \quad f_{j}>0 \text { implies } \beta_{j}=0 .
$$

Since $J^{0}=\left\{j \in J: \alpha_{j}<0<\beta_{j}\right\}$, it follows that

$$
J \supset J_{f} \supset J^{0}, \quad \text { where } J_{f}=\left\{j \in J: f_{j}=0\right\} \text {. }
$$

Note that $J_{f}$ is a proper subset of $J$ since $f_{j} \neq 0$ for at least one $j \in J$. The required property (4.17) (with $x_{j}^{0}=0$ ) holds as soon as $J_{f}=J^{0}$. In particular, one is ready if $J_{f}$ is empty. However, it is quite possible that $J_{f}$ is strictly larger than $J^{0}$, that is, there might exist indices $j \in J$, such that $f_{j}=0$ and either $\alpha_{j}=0$ or $\beta_{j}=0$.

Since $x_{j}^{0}=0(j \in J)$ is the unique solution of (4.2), (4.23), it follows that $x_{j}^{0}=0(j \in J)$ is certainly the unique solution of (4.2), (4.23) but with $J$ replaced by the proper subset $J_{f}$ of $J$. Therefore, our induction assumption implies the existence of numbers $z_{i} \geq 0(i \in$ I) with $z_{i}=0$, for $i \notin I^{0}$, such that the associated numbers $g_{j}=$ $\sum_{i} z_{i} a_{i j}(j \in J)$ satisfy

$$
g_{j}<0 \text { if } \alpha_{j}=0
$$

$g_{j}>0$ if $\beta_{j}=0$;

$g_{j}=0$ if $\alpha_{j}<0<\beta_{j}$,

provided $j \in J_{f}$. Now let $\rho>0$ be small and consider $\eta_{i}=$ $y_{i}+\rho z_{i}(i \in I)$ and the associated numbers $\phi_{j}=\sum_{i} \eta_{i} a_{i j}=f_{j}+\rho g_{j}$ $(j \in J)$. One has $\eta_{i} \geq 0(i \in I)$ and $\eta_{i}=0\left(i \in I^{0}\right)$. Choosing 
$\rho>0$ sufficiently small, one has $f_{j}+\rho g_{j}<0$ when $f_{j}<0$ and $f_{j}+$ $\rho g_{j}>0$ when $f_{j}>0$. Using (4.24) and (4.25), one now easily verifies that (4.16), (4.17) are true for $y_{i}$ replaced by $\eta_{i}(i \in I)$ and $f_{j}$ replaced by $\phi_{j}(j \in J)$. This completes the proof of Theorem 4.15.

4.26. REMARK. Another necessary and sufficient condition for the uniqueness of the solution $x^{0}$ of (4.1), (4.2) would be the existence of a vector $f=\left(f_{1}, \ldots, f_{n}\right)$ as in (4.16) satisfying

$$
\begin{aligned}
x_{j}^{0} & =\alpha_{j} \text { if } f_{j}<0 ; \quad x_{j}^{0}=\beta_{j} \text { if } f_{j}>0 ; \text { and } \\
A^{f} & =\left(a_{i j} ; i \in I^{0} ; f_{j}=0\right) \text { is a matrix of uniqueness. }
\end{aligned}
$$

In particular, $J^{0}$ is a subset of $J^{f}=\left\{i \in J ; f_{j}=0\right\}$. The necessity follows from Theorem 4.15 which shows that one can even attain that $J^{f}=J^{0}$. The sufficiency follows by the same reasoning as before, see 4.21.

4.27. Theorem. Let $x^{0} \in R^{n}$ satisfy

$$
\alpha_{j} \leq x_{j} \leq \beta_{j} \quad(j \in J) .
$$

Let further $J^{0}=\left\{j \in J: \alpha_{j}<x_{j}^{0}<\beta_{j}\right\}$. Then in order that $x^{0}$ be uniquely determined by the vector $A x^{0}$ together with the bounds (4.28), it is necessary and sufficient that: (i) Some linear combination

$$
f_{j}=\sum_{i \in I} y_{i} a_{i j} \quad\left(y_{i} \in R ; i \in I\right)
$$

of the rows of $A$ has property (4.17).

(ii) The submatrix $A^{0}=\left(a_{i j} ; i \in I ; j \in J^{0}\right)$ of $A$ has full rank $\left|J^{0}\right|$. Equivalently, if $A z=0$ and $z_{j}=0$ for $j \notin J^{0}$ then $z=0$.

4.30. Proof. Apply Theorem 4.15 with $A$ replaced by $B=\left(\begin{array}{c}A \\ -A\end{array}\right)$ and $b$ replaced by $\left(\begin{array}{c}b \\ -b\end{array}\right)$ with $b=A x^{0}$. Presently, $I^{0}=I$. The matrix $B^{0}$ associated to $B$ is a matrix of uniqueness if and only if $A^{0}$ has rank $\left|J^{0}\right|$ (see 4.10).

4.31. Corollary. Let $x^{0} \in R^{n}$ satisfy

$$
x_{j}^{0} \in\left\{\alpha_{j}, \beta_{j}\right\}, \quad \text { for all } j \in J .
$$

Then in order that $x^{0}$ be unquely determiined by the vector $A x^{0}$ together with the conditions (4.28), it is necessary and sufficient that there exists a linear combination as in (4.29) such that

$$
f_{j}<0 \text { if } x_{j}=\alpha_{j} ; \quad f_{j}>0 \text { if } x_{j}=\beta_{j} .
$$

In particular, $f_{j} \neq 0$ for all $j \in J$. 
4.34. REMARK. Theorem 4.27 and Corollary 4.31 remain valid when $I$ is infinite, provided we add to (4.29) the condition that $y_{i}=0$ for all but finitely many $i \in I$. In such a situation, the condition $A x=A x^{0}$ amounts to an infinite system of equalities.

It suffices to show that (i), (ii) are necessary. For each finite subset $H$ of $I$, let $D_{H}$ be the closed convex set consisting of all $x \in R^{n}$ satisfying (4.28) and $\sum_{j} a_{i j} x_{j}=\sum_{j} a_{i j} x_{j}^{0}$, for each $i \in H$. Assuming that $\bigcap_{H} D_{H}=\left\{x^{0}\right\}$, it suffices to show that $H$ exists with $D_{H}=\left\{x^{0}\right\}$, that is, $\operatorname{dim} D_{H}=0$. Let $H^{*}$ be such that $\operatorname{dim} D_{H^{*}}=\inf f_{H} \operatorname{dim} D_{H}$. One easily verifies that $D_{H}=D_{H^{*}}$ for each $H \supset H^{*}$ implying that $D_{H^{*}}=\bigcap_{H} D_{H}=\left\{x^{0}\right\}$. Consequently, if $x^{0}$ is a vector of uniqueness relative to $(4.1),(4.2)$ then also relative to (4.2) together with a suitably chosen finite subsystem of (4.1).

4.35. Remark. Relative to the prescribed $m \times n$ matrix $A=\left(a_{i j}\right)$ and extended real numbers $\alpha_{j}, \beta_{j}\left(\alpha_{j}<\beta_{j} ; j \in J\right)$, let us say that $x^{0} \in R^{n}$ is a vector of uniqueness if it satisfies (4.28) and moreover, there is no other vector $x$ satisfying (4.28) and $A x=A x^{0}$. If, in addition, $x^{0}$ satisfies (4.32), then we will say that $x^{0}$ is a special vector (of uniqueness). Corollary 4.31 amounts to a recipe for finding all such special vectors $x$. Namely, define $x_{j}=\alpha_{j}$ if $f_{j}<0 ; x_{j}=\beta_{j}$ if $f_{j}>0$ with $f$ as any linear combination, as in (4.29), such that $f_{j} \neq 0$ for all $j \in J ;\left(\alpha_{j}\right.$ finite if $f_{j}<0 ; \beta_{j}$ finite if $\left.f_{j}>0\right)$. In many applications, such a sequence $\left\{f_{j}\right\}$ has only a limited number of changes of sign thus severely limiting the possible special vectors $x^{0}$.

4.36. As is often true, suppose there exists $z \in R^{m}$ such that

$$
w_{j}:=\sum_{i \in I} z_{i} a_{i j}>0 \text { for all } j \in J .
$$

Then an equivalent recipe would be to define

$$
x_{j}=\alpha_{j} \text { if } f_{j}<0 ; \quad x_{j}=\beta_{j} \text { if } f_{j} \geq 0, \quad \text { for all } j \in J .
$$

with $f$ as any linear combination, as in (4.29), such that $\alpha_{j}$ is finite when $f_{j}<0 ; \beta_{j}$ is finite with $f_{j} \geq 0$.

We already know that every special vector (of uniqueness) can be realized in this manner even with $f_{j} \neq 0$ for all $j \in J$. That every vector of the type (4.38) (with $f$ as indicated) is a vector of uniqueness can be seen either by an easy direct proof (analogous to the proof 
of Lemma 2.14) or else by introducing $\phi_{j}=f_{j}+\rho w_{j}(j \in J)$ with $\rho>0$ so small that $f_{j}<0$ implies $\left.\phi_{j}<0\right)$.

4.39. Measures determined by their marginals. Here, we change some of the previous notations. Let $X$ be a fixed finite non-empty set and let $\pi_{j}: X \rightarrow Y_{j}(j \in J)$ be given mappings, (where $J$ can have any cardinality). Let further $-\infty \leq \alpha(x)<\beta(x) \leq+\infty,(x \in X)$, be given extended real numbers and let

(4.40) $M_{0}=\{\phi: X \rightarrow R$ such that

$$
\alpha(x) \leq \phi(x) \leq \beta(x), \text { for all } x \in X\} .
$$

Each such $\phi \in M_{0}$ can also be regarded as a finite signed measure $\mu$ on $X$ with mass $\mu(\{x\})=\phi(x)$ at $x \in X$. The associated $\pi_{j}$-marginal (of $\phi$ or $\mu$ ) is given by

$$
g_{j}(y)=\left(\pi_{j} \mu\right)(\{y\})=\sum\left\{\phi(x): \pi_{j} x=y\right\}, \quad\left(y \in Y_{j} ; j \in J\right) .
$$

The following result characterizes the $\phi \in M_{0}$ which are uniquely determined by these marginals.

4.42. Theorem. Suppose $X$ is finite. Let $\phi \in M_{0}$ and put

$$
X^{0}=\{x \in X: \alpha(x)<\phi(x)<\beta(x)\} .
$$

In order that there exists no other $\psi \in M_{0}$ having the same marginals as $\phi$, it is necessary and sufficient that:

(i) There does not exist any non-zero function $h: X \rightarrow R$ which is supported by $X^{0}$ (that is, $h(x)=0$ if $x \notin X^{0}$ ) and has all its marginals equal to zero.

(ii) There exists a function $f: X \rightarrow R$ of the special form

$$
f(x)=\sum_{j \in J} f_{j}\left(\pi_{j} x\right) \text { with } f_{j}: Y_{j} \rightarrow R(j \in J),
$$

with only finitely many non-zero functions $f_{j}$, and such that

$$
\begin{gathered}
f(x)<0 \text { if } \phi(x)=\alpha(x) ; \quad f(x)>0 \text { if } \phi(x)=\beta(x) \\
f(x)=0 \text { if } x \in X^{0}
\end{gathered}
$$

4.46. Proof. Apply Theorem 4.27, extended as in Remark 4.34. In the previous discussion, replace $J=\{1, \ldots, n\}$ by $X ; J^{0}$ by $X^{0}$; $j$ by $x ; x_{j}$ by $\phi(x) ; \alpha_{j}$ by $\alpha(x)$ and $\beta_{j}$ by $\beta(x)$. And finally replace the index set $I$ by the set of pairs $(j, y)$ where $j \in J$ and $y \in Y_{j}$. In view of (4.41), the condition that $\phi$ and $\psi$ have the 
same marginals is analogous to $A x^{0}=A x$ with $A$ as a matrix (one row for each choice of $j \in J$ and $y \in Y_{j}$; if $J$ is infinite then there are infinity many rows). Clearly, the matrix element $a_{(j, y), x}$ which corresponds to the pair of indices $(j, y) \in I$ and $x \in X$, equals 1 if $\pi_{j} x=y$ and equals 0 , otherwise. Therefore, (4.29) now takes the form (4.44), while condition (4.17) of Theorem 4.27 takes the form (4.45). Finally, the present condition (i) is precisely condition (ii) of Theorem 4.27.

4.47. Additive sets. For convenience assume that $\alpha(x), \beta(x)$ $(x \in X)$ are finite. Call $\phi: X \rightarrow R$ special if $\phi(x) \in\{\alpha(x), \beta(x)\}$, for all $x \in X$, and further no other $\psi \in M_{0}$ has the same marginals as $\phi$. From Theorem 4.42, $\phi: X \rightarrow R$ is special if and only if

$$
\phi(x)=\alpha(x) \text { if } f(x)<0 ; \quad \phi(x)=\beta(x) \text { if } f(x)>0,
$$

for some $f: Y \rightarrow R$ of the form (4.44) and such that $f(x) \neq 0$ for all $x \in X$.

As an equivalent criterion, $\phi: X \rightarrow R$ is special if and only if

$$
\phi(x)=\alpha(x) \text { if } f(x)<0 ; \quad \phi(x)=\beta(x) \text { if } f(x) \geq 0,
$$

for some $f: X \rightarrow R$ of the form (4.44). This follows from Remark 4.36. After all, $\sum_{y} a_{(j, y), x}=1$, for all $j$ and $x$ so that condition (4.37) is satisfied.

The case $\alpha(x)=0 ; \beta(x)=q(x)$ can be stated as follows, showing that the converse of Lemma 2.14 holds when $X$ is finite. For the case that $X$ and the $\pi_{j}$ are as in Example 2.7, the result is largely due to Fishburn et al. [2].

4.50. Theorem. Let $S$ be a subset of $X$, where $X$ is finite. Then the following are equivalent.

(i) $S$ is a set of uniqueness.

(ii) $S$ is additive, that is, $S=\{x \in X: f(x) \geq 0\}$, for some $f \in F(S)$, see Definition 2.11 .

(iii) $S=\{x \in X: f(x) \geq 0\}$ for some $f: X \rightarrow R$ of the form $f(x)=\sum_{j \in J} f_{j}\left(\pi_{j} x\right)$, with all but finitely many $f_{j}: Y_{j} \rightarrow R$ equal to zero.

(iv) Same as (iii), except that we require in addition that $f(x) \neq 0$, for all $x \in X$.

4.51. REMARK. The reader should keep in mind that, when $X$ is finite, there may exist subsets $S$ of $X$ such that, for no other set $T$ 
does $1_{T} d \lambda$ have the same projections as $1_{S} d \lambda$, while nevertheless $\phi=1_{S}$ is not a (special) vector of uniqueness; (this cannot happen in the situation of Example 2.6, see [1], [4]). From Lemma 2.14, such a set $S$ cannot possibly be additive. Natually, the explanation is that in such a situation there exists a function $\psi: X \rightarrow R$, not of the form $\psi(x) \in\{0,1\}$, such that $\psi d \lambda$ has the same projections as $1_{S} d \lambda$. An example of such a set $S$ was given in [2]. It uses the structure of Example 2.7 with $n=3 ;|S|=66$ and $X=\{1,2,3,4,5\}^{3}$ thus $|X|=125$.

\section{REFERENCES}

[1] P. C. Fishburn, J. C. Lagarias, J. A. Reeds, and L. A. Shepp, Sets uniquely determined by projections on axes I. Continuous case, SIAM J. Appl. Math., 50 (1990), 286-306.

[2] _ Sets uniquely determined by projections on axes. II. Discrete case, preprint.

[3] D. Gale, The Theory of Linear Economic Models, McGraw-Hill Book Co., New York, 1960.

[4] S. Gutmann, J. H. B. Kemperman, and L. A. Shepp, Existence of probability measures with given marginals, Ann. Probab., to appear.

[5] J. H. B. Kemperman, On the lack of uniqueness when reconstructing a set from finitely many central projections, in preparation.

[6] __, On sets that are uniquely determined by a restricted set of integrals, Trans. Amer. Math. Soc., 322 (1990), 417- 458.

[7] A. Kuba and A. Volcic, Characterization of measurable plan sets, which are reconstructible from their two projections, Inverse Problems, 4 (1988), 513-527.

[8] G. G. Lorentz, A problem of plane measures, Amer. J. Math., 71 (1949), 417 426.

[9] A. Volcic, On a class of plane sets, which can be reconstructed from two Steiner symmetrizations, manuscript.

Received November 15,1988 . This research was supported in part by the National Science Foundation Grant DMS-8802557.

RUTGERS UNIVERSITY

New BRUnSWICK, NJ 08903 



\section{PACIFIC JOURNAL OF MATHEMATICS EDITORS}

\author{
V. S. VARADARAJAN \\ (Managing Editor) \\ University of California \\ Los Angeles, CA 90024-1555-05 \\ Herbert Clemens \\ University of Utah \\ Salt Lake City, UT 84112 \\ THOMAS ENRIGHT \\ University of California, San Diego \\ La Jolla, CA 92093
}

R. FINN

Stanford University

Stanford, CA 94305

HeRmann FlaschKa

University of Arizona

Tucson, AZ 85721

VAUghan F. R. Jones

University of California

Berkeley, CA 94720

SteVEN KeRCKHOFF

Stanford University

Stanford, CA 94305

\section{C. MOORE}

University of California

Berkeley, CA 94720

MARTIN SCHARLEMANN

University of California

Santa Barbara, CA 93106

HAROLD STARK

University of California, San Diego

La Jolla, CA 92093

\section{ASSOCIATE EDITORS \\ R. ARENS \\ E. F. BECKENBACH \\ B. H. NeUmanN \\ F. WoLF \\ (1904-1989) \\ K. Yoshida (1906-1982)}

\section{SUPPORTING INSTITUTIONS}

UNIVERSITY OF ARIZONA

UNIVERSITY OF BRITISH COLUMBIA

CALIFORNIA INSTITUTE OF TECHNOLOGY

UNIVERSITY OF CALIFORNIA

MONTANA STATE UNIVERSITY

UNIVERSITY OF NEVADA, RENO

NEW MEXICO STATE UNIVERSITY

OREGON STATE UNIVERSITY
UNIVERSITY OF OREGON

UNIVERSITY OF SOUTHERN CALIFORNIA

STANFORD UNIVERSITY

UNIVERSITY OF HAWAII

UNIVERSITY OF TOKYO

UNIVERSITY OF UTAH

WASHINGTON STATE UNIVERSITY

UNIVERSITY OF WASHINGTON

The Supporting Institutions listed above contribute to the cost of publication of this Journal, but they are not owners or publishers and have no responsibility for its content or policies.

Mathematical papers intended for publication in the Pacific Journal of Mathematics should be in typed form or offset-reproduced (not dittoed), double spaced with large margins. Please do not use built up fractions in the text of the manuscript. However, you may use them in the displayed equations. Underline Greek letters in red, German in green, and script in blue. The first paragraph must be capable of being used separately as a synopsis of the entire paper. In particular it should contain no bibliographic references. Please propose a heading for the odd numbered pages of less than 35 characters. Manuscripts, in triplicate, may be sent to any one of the editors. Please classify according to the 1991 Mathematics Subject Classification scheme which can be found in the December index volumes of Mathematical Reviews. Supply name and address of author to whom proofs should be sent. All other communications should be addressed to the managing editor, or Elaine Barth, University of California, Los Angeles, California 90024-1555-05.

There are page-charges associated with articles appearing in the Pacific Journal of Mathematics. These charges are expected to be paid by the author's University, Government Agency or Company. If the author or authors do not have access to such Institutional support these charges are waived. Single authors will receive 50 free reprints; joint authors will receive a total of 100 free reprints. Additional copies may be obtained at cost in multiples of 50 .

The Pacific Journal of Mathematics (ISSN 0030-8730) is published monthly except for July and August. Regular subscription rate: $\$ 190.00$ a year (10 issues). Special rate: $\$ 95.00$ a year to individual members of supporting institutions.

Subscriptions, orders for numbers issued in the last three calendar years, and changes of address should be sent to Pacific Journal of Mathematics, P.O. Box 969, Carmel Valley, CA 93924, U.S.A. Old back numbers obtainable from Kraus Periodicals Co., Route 100, Millwood, NY 10546.

The Pacific Journal of Mathematics at P.O. Box 969, Carmel Valley, CA 93924 (ISSN 0030-8730) is published monthly except for July and August. Second-class postage paid at Carmel Valley, California 93924, and additional mailing offices. Postmaster: send address changes to Pacific Journal of Mathématics, P.O. Box 969, Carmel Valley, CA 93924.

PUBLISHED BY PACIFIC JOURNAL OF MATHEMATICS, A NON-PROFIT CORPORATION Copyright (C) 1991 by Pacific Journal of Mathematics 


\section{Pacific Journal of Mathematics}

Vol. 148, No. $2 \quad$ April, 1991

Yuri A. Abramovich, Operators preserving disjointness on rearrangement

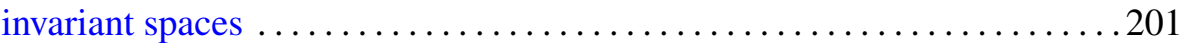

Andrew French Acker and Kirk Lancaster, Existence and geometry of a free boundary problem for the heat equation .................. 207

So-Chin Chen, Real analytic regularity of the Szegő projection on circular

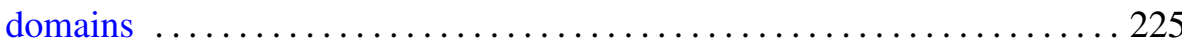

Chen-Lian Chuang, An independence property of central polynomials . . . 237

Peter Larkin Duren and M. Schiffer, Robin functions and energy

functionals of multiply connected domains $\ldots \ldots \ldots \ldots \ldots \ldots \ldots \ldots . \ldots 251$

Johan Henricus Bernardus Kemperman, Sets of uniqueness and systems

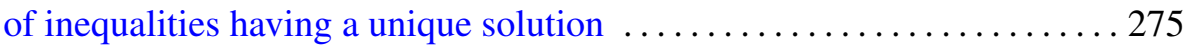

Ka-Lam Kueh, Fourier coefficients of nonholomorphic modular forms and sums of Kloosterman sums . .......................... 303

Gerard J. Murphy, Ordered groups and crossed products of $C^{*}$-algebras . . 319

You-Qiang Wang, The $p$-parts of Brauer character degrees in $p$-solvable

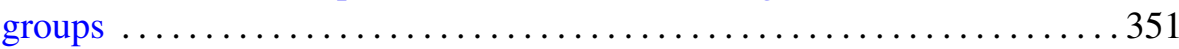

Hidenobu Yoshida, Harmonic majorization of a subharmonic function on a cone or on a cylinder ........................................ 369 